\title{
A PRELIMINARY MECHANICAL DESIGN OF A 500MHZ ACCELERATING CAVITY WITH DAMPED HIGH ORDER MODES
}

\author{
A. Daly, D.M. Dykes, C.L. Hodgkinson and P.A. McIntosh, CLRC, Daresbury Laboratory
}

\begin{abstract}
Daresbury Laboratory is involved in a collaboration with BESSY, DELTA, MAX-Lab and NTHU to develop a normally conducting $500 \mathrm{MHz}$ accelerating cavity for electron storage rings with significantly damped high order modes (HOM's). This paper will describe some of the mechanical engineering challenges facing the project and suggest possible solutions. Finite element analysis using the high frequency electromagnetic module in ANSYS has been used to calculate the thermal and structural stresses. These results have then been used to determine the cooling requirements during operation at $100 \mathrm{~kW}$. Methods of fabrication have been evaluated with a view to minimising cost and complexity. A preliminary investigation into various tuning techniques has also been carried out.
\end{abstract}

\section{INTRODUCTION}

The result of this project is to develop a normally conducting $500 \mathrm{MHz}$ accelerating cavity for $3^{\text {rd }}$ generation electron storage rings. Many storage rings worldwide operate at this frequency and therefore the cavity has the potential for widespread application. In the case of the Synchrotron Radiation Source (SRS) at Daresbury Laboratory, there is a proposal to move to higher operating currents $300 \mathrm{~mA}$ and above, this would be difficult with the four current $500 \mathrm{MHz}$ cavities due to cavity induced beam instabilities. By replacing the current cavities with two of the new cavities beam currents up to $500 \mathrm{~mA}$ may be achieved and two more straights will become free for insertion devices.

There have been two RF designs considered a spherical cavity [1] proposed by Daresbury Laboratory and a cylindrical cavity [2] proposed by BESSY. Daresbury Laboratory has led the design of the spherical cavity and as a result this paper will concentrate on that design although some results from the preliminary thermal analysis of the cylindrical cavity will be presented.

\section{THE MECHANICAL DESIGN}

The detail design of the cavity is dependant on many factors, including cooling requirements and manufacturing technique employed. In order to determine some of these requirements basic geometry of the cavity is required to allow initial thermal and structural analysis to be carried out. The cavity is a spherical, re-entrant cavity and incorporates 3 equi-spaced ridged waveguide tapers, with circular waveguide to coaxial transmission to couple out the HOM power to the HOM loads.

\subsection{Geometry}

Figure 1 shows the EU cavity geometry used for the preliminary analysis.

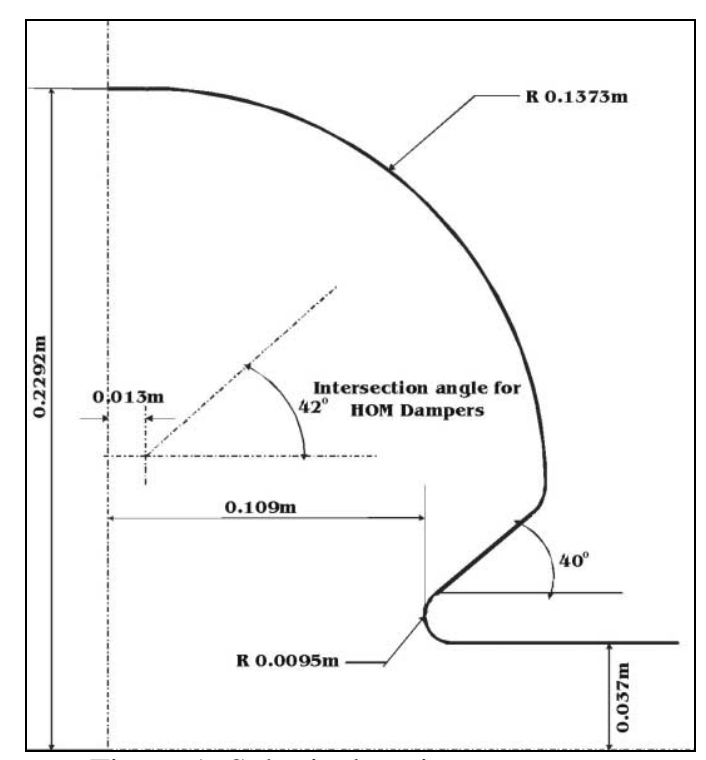

Figure 1: Spherical cavity geometry

The internal geometry was given a shell of $5 \mathrm{~mm}$ thick Oxygen Free High Conductivity (OFHC) Copper. Although not a complete engineering design, this is ideal for a thermal analysis. The results of which will significantly aid the mechanical engineering design process.

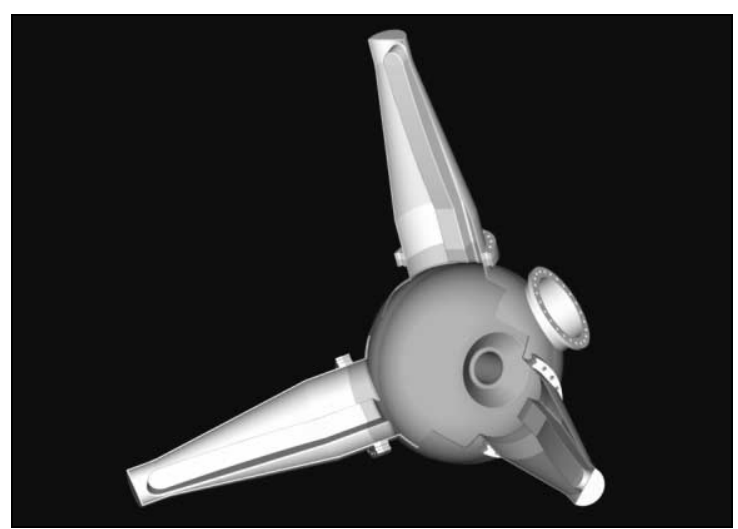

Figure 2: Cut away model of the cavity

\subsection{Tuner Design}

Three methods of tuning room temperature cavities have been used with much success. Controlling the 
temperature of the cavity, squeezing the cavity longitudinally and the use of a water-cooled plunger. Two of theses three methods are essential to ensure the cavity can operate in a HOM free area on its fundamental frequency. Two solutions were considered.

Temperature variation in combination with longitudinally squeezing the cavity is used at ELETTRA [3]. The system is complex and the short timescales for the EU cavity make the development of the system for this project difficult.

Temperature variation combined with a water-cooled plunger has been used reliably for over 20 years at the SRS [4] and requires little development to integrate the current SRS plunger into the EU cavity design. Therefore it was decided to proceed with this method of tuning.

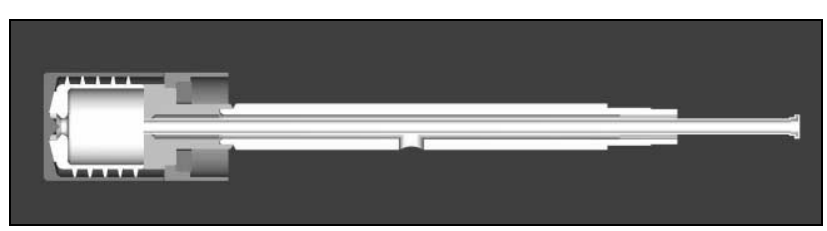

Figure 3: SRS tuner

\subsection{Manufacturing Techniques}

There are several techniques available for the production of RF cavities and all have been briefly investigated.

The SRS cavities were manufactured by electroforming. This is a specialist fabrication process during which material is electro deposited on a mandrel which is subsequently removed. The mandrel is replica of the internal profile of the component to be fabricated, therefore complex internal geometries can be reproduced with tolerances of up to $1 \mu \mathrm{m}$. Another advantage is multiple layers can be molecularly bonded together, for example a jacket can be bonded on to cover water-cooling channels. However, this process is costly as the mandrel is either destroyed or significant development work is required to produce a collapsible mandrel. Also the deposition rate is slow approximately $0.03 \mathrm{~mm}$ per hour.

An alternative approach is to conventionally manufacture and fabricate the cavity. A range of techniques must be employed to get the best results for example e-b welding, TIG welding and brazing. This is a more cost effective method of manufacture, however the design of the fabrication and in particular the sequence of joining must be carefully considered to ensure the dimensional accuracy and vacuum integrity of the cavity.

The final technique under consideration is to manufacture the cavity from a bulk material, for example aluminium and coat the internal surfaces with a consistent $50 \mu \mathrm{m}$ layer of OFHC Copper. The lower cost of the material would allow an increased thickness and simplify the design of the support structure required for the cavity and HOM dampers. The feasibility of this solution needs further investigation as the techniques employed are usually 'line of sight' processes and due to the complex internal geometry of the cavity a consistent coating may not be possible to achieve.

\section{FINITE ELEMENT ANALYSIS}

Finite element analysis was carried out using the ANSYS software. ANSYS has an electromagnetic module, which greatly simplifies the transition from RF design to mechanical design, as the same mesh can be used throughout [5]. To reduce processing time the analysis is carried out on a specially prepared $1 / 6$ model of the cavity with detail features such as weld preparations and flanges removed. It is critical to select a section of the cavity with the major features present, such as a HOM damper and input coupler. It has been assumed that the cavity will be manufactured in OFHC Copper and table 1 shows the critical properties used in the analysis.

Table 1: OFHC Material Properties

\begin{tabular}{|l|c|}
\hline Young's Modulus (GPa) & 126 \\
\hline Coefficient of Thermal Expansion $(/ \mathrm{K})$ & $1.67 \times 10^{-5}$ \\
\hline Thermal Conductivity (W/mK) & 392 \\
\hline $0.2 \%$ Proof Stress $(\mathrm{MPa})$ & 124 \\
\hline Relative Permittivity & 1 \\
\hline Relative Permeability & 1 \\
\hline
\end{tabular}

\subsection{High Frequency Analysis}

The cavity vacuum space is modelled with high frequency solid elements and an electric wall boundary condition applied to the internal faces of the cavity. The analysis calculated the fundamental frequency of the cavity to be $489 \mathrm{MHz}$, an $11 \mathrm{MHz}(2 \%)$ discrepancy. There are two possible causes of this small error. One cause is as only a $1 / 6$ of the cavity is modelled the large ports have the effect of reducing the calculated fundamental frequency. Another is due to the refinement of the mesh in critical areas such as around the nose cones and HOM ports. Due to the break in symmetry the magnetic field on axis is not zero and the $\mathrm{H}$-field plot shown in figure 4 can be used to evaluate the refinement of the mesh. The curve should be smooth and additional refinement is required.

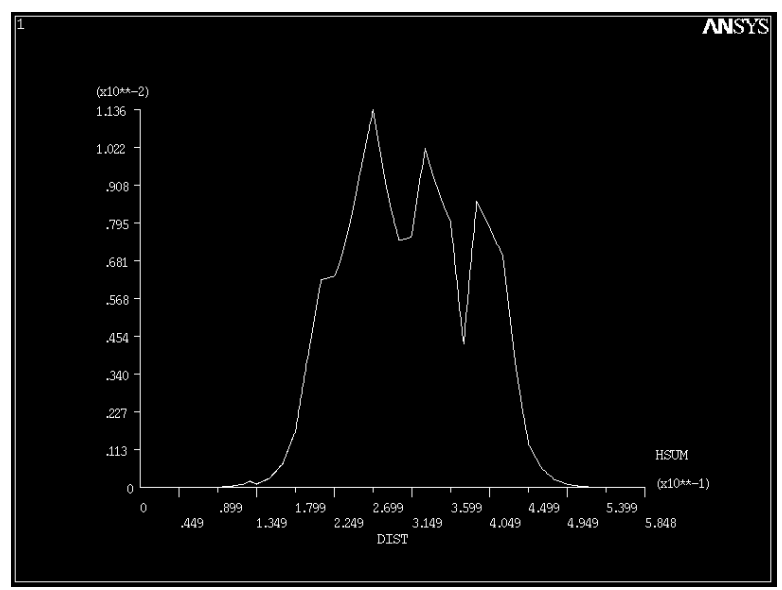

Figure 4: H-field along beam axis 


\subsection{Thermal Analysis}

The currents induced by the surface magnetic field can then be used to determine the power dissipation on the internal surfaces of the cavity. A macro provided by Neal Hartman of Lawrence Berkeley National Laboratory (LBNL) carries out the calculation for each surface element. The maximum heat fluxes occur where the HOM dampers and input couplers join the cavity body. The analysis determined a maximum flux of $48 \mathrm{~W} / \mathrm{cm}^{2}$ for an input power of $100 \mathrm{~kW}$. As the analysis was carried out to determine the best location for cooling channels a convection boundary condition was applied to all external surfaces of the cavity to simulate $25^{\circ} \mathrm{C}$ water. The maximum temperature rise is $44.6^{\circ} \mathrm{C}$ at the hot spot as shown in figure 5.

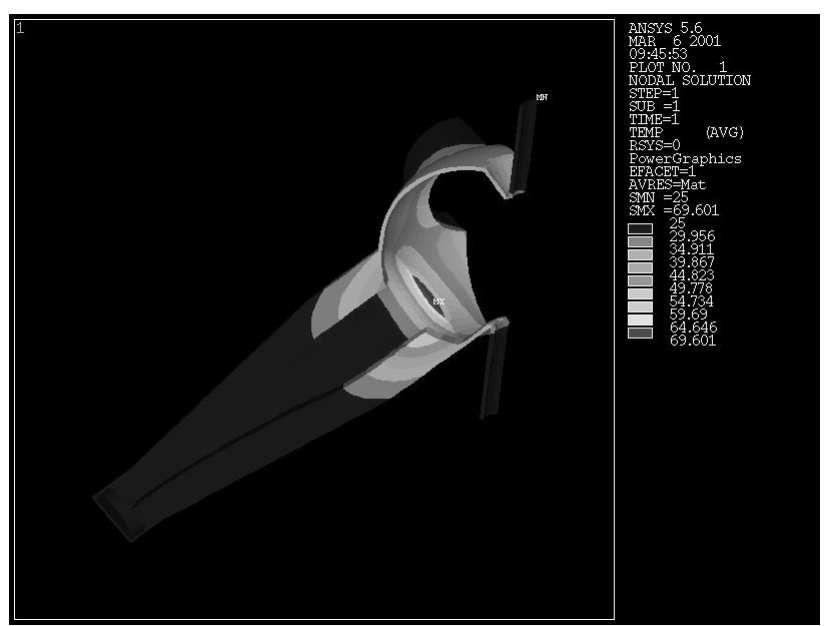

Figure 5: Temperature rise

\subsection{Structural Analysis}

As the cavity is currently a thin shell to model cooling atmospheric pressure was not included in the analysis. The stress induced in the cavity by thermal loading is approximately $60 \mathrm{MPa}$, comfortably within the safe working limit of $124 \mathrm{MPa}$ for OFHC Copper.

Figure 6 shows the displacement of the cavity. The maximum displacement is $0.37 \mathrm{~mm}$ and occurs at the end of the HOM damper. The design of the support structure for the cavity must be designed carefully to avoid over constraining the port and inducing unnecessary additional stresses. For this type of cavity it is essential to keep the movement of the nose cones to a minimum, as this has the greatest effect on the frequency of the cavity. The analysis predicted a nose-cone movement of $0.08 \mathrm{~mm}$ into the cavity. This would result in a frequency shift of approximately $256 \mathrm{kHz}$.

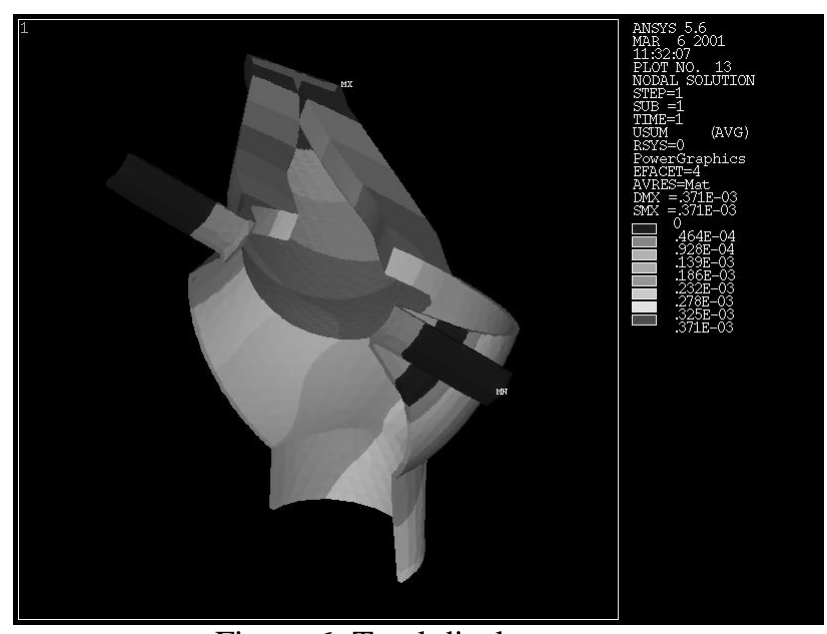

Figure 6: Total displacement

\section{CONCLUSIONS AND FURTHER WORK}

The preliminary work carried out so far clearly demonstrates the feasibility of the spherical cavity design. It has been proposed that both the cylindrical and spherical designs are developed to the detailed mechanical design stage and costed. At this point a decision will be taken on which cavity to proceed with.

A preliminary FEA study of the cylindrical cavity has been carried out and the results obtained for $100 \mathrm{~kW}$ show a maximum heat flux of $62 \mathrm{~W} / \mathrm{cm}^{2}$. Slightly higher than the figure of $48 \mathrm{~W} / \mathrm{cm}^{2}$ calculated for the spherical cavity. Both cavities are feasible to manufacture, however the simpler geometry of the cylindrical cavity may reduce some of the engineering challenges.

Daresbury Laboratory will continue to carry out FEA and design the cooling for both cavity designs and continue with the detailed mechanical design of the spherical cavity.

\section{ACKNOWLEDGEMENTS}

The authors would like to thank Neal Hartman of LBNL, USA for passing on his knowledge and assistance in using ANSYS to determine RF heating and for the use of macros required to convert magnetic field into surface power loss.

\section{REFERENCES}

[1] P.A. McIntosh et al, "A Possible Upgrade for the SRS RF System", these proceedings.

[2] F. Marhauser et al, "HOM Damped 500MHz Cavity Design for $3^{\text {rd }}$ Generation SR Sources", these proceedings.

[3] A.Fabris et al, 'Characterisation of the Low Level System of the ELETTRA RF Plants', EPAC'96 Proceedings, p1917.

[4] P.A.McIntosh et al, 'Temperature dependant High Order Modes (HOM) in the SRS Cavities', EPAC'96 Proceedings, p1961.

[5] N. Hartman et al, 'Electromagnetic, Thermal, and Structural Analysis of RF Cavities Using ANSYS', these proceedings. 